Darüber hinaus gab es während der gesamten Zeit der Konferenz eine Freiluft-Installation am Heldenplatz („,The Journey against sex trafficking“) von der Oscar-Preisträgerin Emma Thompson. In sieben von verschiedenen Künstlern ${ }^{2}$ gestalteten Containern wird der Leidensweg einer jungen Frau beschrieben, die ihr „Glück“ im Ausland suchen will und in die Hände von Menschenhändlern gerät. Die Container tragen Namen wie „Hope“, „Uniform“, „Bedroom“ oder „Customer“. Die Installation war bereits im letzten Jahr mit großem Erfolg in New York und London zu sehen und wird weiter rund um den Golobus reisen.

Abgerundet und beendet wurde die Tagung schließlich durch eine Podiumsdiskussion, in der sich Helga Konrad ${ }^{3}$, Andrew Dismore ${ }^{4}$ sowie Chris Smith ${ }^{5}$ mit der Rolle der Parlamentarier bei der Bekämpfung des Menschenhandels auseinandersetzten.

Mehr Informationen zu dem Wiener Forum gegen Menschenhandel sowie das 19 Seiten starke Draft-summery „The appropriate legal responses to combating trafficking in persons, A handbook for Par- liamentarians" kann man kostenlos unter www.ungift.org oder viennaforum@unov.org erhalten.

Ina Hunecke ist wissenschaftliche Mitarbeiterin an der CAU Kiel

Fußnoten:

1 Die UN-GIFT wurde im März 2007 gemeinsam vom Büro der Vereinten Nationen für Drogen- und Verbrechensbekämpfung (UNODC), der Internationalen Arbeitsorganisation (ILO), der Internationalen Organisation für Migration (IOM), dem Kinderhilfswerk der Vereinten Nationen (UNICEF), dem Hohen Kommissar der Vereinten Nationen für Menschenrechte (UNHCR) und der Organisation für Sicherheit und Zusammenarbeit in Europa (OSZE) gegründet. Innerhalb dieser Initiative bündelt die UNO die Bemühungen mehrerer ihrer Organisationen, die sich bisher parallel mit Teilaspekten dieses Themas befasst haben.

2 z.B. Oscar-Gewinner für Filmdesign: Michael Howells, Oscar-Gewinner für Kostüme: Sandy Powell und Gewinner des Turner-Preises: Anish Kapoor.

3 ehemalige OSZE-Sonderbeauftragte zur Bekämpfung des Menschenhandels

4 Abgeordneter des britischen Unterhauses

5 Mitglied des US-Kongresses

\title{
Die Verschärfung des sexuellen Missbrauchs von Jugendlichen und die Kriminalisierung des Besitzes von sog. Jugendpornografie*
}

Monika Frommel

Bis 1994 galt im Bereich der alten Bundesrepublik noch eine Jugendschutznorm des $\$ 175$ (Homosexuelle Handlungen). In den neuen Bundesländern galt bis zu diesem Zeitpunkt aufgrund des Einigungsvertrages noch der alte $\$ 149$ StGB-DDR (Einfacher Missbrauch) weiter. Dieser sah ein einheitliches Schutzalter für homo- und heterosexuelle Handlungen vor:

\149. Sexueller Mißbrauch von Jugendlichen. (1) Ein Erwachsener, der einen Jugendlichen zwischen vierzehn und sechzehn Jahren unter Ausnutzung der moralischen Unreife durch Geschenke, Versprechen von Vorteilen oder in ähnlicher Weise dazu mißbraucht, mit ihm Geschlechtsverkehr auszuüben oder geschlechtsverkehrsähnliche Handlungen vorzunehmen, wird mit Freiheitsstrafe bis zu zwei Jahren oder mit Verurteilung auf Bewährung bestraft. (2) Die Strafverfolgung verjährt in zwei Jahren.

1993 einigte man sich auf einen Kompromiss. Die neue einheitlich Strafbestimmung zum Schutz Jugendlicher des $\ 182$ StGB sah ein Schutzalter von 16 und 18 Jahre vor, Täter kann aber de lege lata nur eine Person über 18 Jahre sein. Die geplante Veränderung dieses Kompromisses und die gleichzeitig geplante Verschärfung des absoluten Verbotes der Kinderpornografie auf alle Formen der „Jugendpornografie“ (Definition völlig unklar) verändert die Struktur der bislang geltenden Straftatbestände erheblich und war deshalb bereits mehrfach Gegenstand von Artikeln der NK (NK 4- 2006 und NK 1 2007).

Am 26. August 2006 beschloss das Bundeskabinett - angeblich in Umsetzung europäischer Vorgaben - eine Reihe von Gesetzen zu ändern, da die Schutzaltersgrenzen angeblich in sich widersprüchlich und uneinheitlich seien. Im Vordergrund stand dabei neben einer
Verschärfung des $\$ 182$ StGB eine erhebliche Ausweitung und Erweiterung des Verbotes der Kinderpornografie, die nun auf Jugendpornografie (Schutzalter 18 Jahre) erweitert werden soll. Wie eine solche Erweiterung praktisch aussehen soll, ist unklar, da mit dem Wegfall des Bezugs auf den verbotenen sexuellen Missbrauch von Kindern gem. $\$ 176$ StGB sowohl die Tathandlung als auch das Schutzgut völlig unklar wird. Denn das verbot von Pornografie dienst dem Jugendschutz. Jugendliche sollen davon abgehalten werden derartige Darstellungen anzuschauen. Bei der auch für Erwachsene verbotene Gewalt- und Kinderpornografie hat man sich geeinigt, dass derartige Verbote hinzunehmen seien, da schließlich auch sexuelle Kontakte mit Kindern und gewalttätige sexuelle Handlungen verboten seien. Aber das betrachten von Darstellungen Jugendlicher ist Erwachsenen erlaubt. Wieso versucht die Gesetzgebung daher nicht den Datenschutz von Jugendlichen zu verbessern. Denn es sind nicht nur sexuelle Darstellungen im Netz, welche für die weitere Entwicklung dieser dargestellten Jugendlichen schädlich sein können. Bestraft werden Verstöße gegen das informelle Selbstbestimmungsrecht auch de lege lata schon durch das Bundesdatenschutzgesetz. Aber die Umsetzung ist mangelhaft. Zustimmen können Jugendliche nämlich solchen Darstellungen nicht, da sie noch nicht voll geschäftsfähig sind. Man könnte daher Personen unter 18 Jahren schützen. Aber nicht über ein Verbot, das sich gegen erwachsene Betrachter richtet. Von einer solchen Strafgesetzgebung haben die Jugendliche wenig. Der Gesetzesentwurf ist im Dezember 2007 vorerst zurückgezogen worden. Aber wenn die öffentliche Kritik verstummt, ist mit einer Neuauflage zu rechnen. 


\section{Einwände gegen die Änderungen}

Einen besseren Schutz gegen Gefährdungen unterhalb der Schwelle der „Ausbeutung“ - denn diese ist auch zu Lasten von Erwachsenen als ausbeuterische Prostitution nach $\ 180$ a StGB strafbar - könnten Jugendliche insbesondere bei der Herstellung und dem Vertrieb von Pornografie de lege ferenda erhalten, wenn man die veraltete Konzeption der $\mathbb{\int} 180$ Abs. 3, 180 a Abs. 2 Nr. 1 (Verbot der Wohnungsgewährung zum Zwecke der Prostitution an unter 18 Jährige) den modernen Gegebenheiten anpassen würde und bestimmte Formen der Kommerzialisierung der Sexualität Jugendlicher auch strafrechtlich untersagen würde.

Dies macht aber nur Sinn, wenn dann die unbedenklichen Formen der Prostitution und der Sexarbeit insgesamt konsequent mit zivilrechtlichen Schutznormen zugunsten der dort Beschäftigten ausgestattet würden. Hier scheut die Bundesregierung einen Konflikt mit den Ländern und den Kommunen, die sich weigern von ihren jeweiligen Kompetenzen den Arbeitsplatz von Prostituierten menschenwürdiger zu gestalten angemessen Gebrauch zu machen. Die Flucht ins Strafrecht ist aber kontraproduktiv.

Sofern sich die Bundesregierung auf EU-Rahmenbeschlüsse, die sie angeblich umsetzen müsse, beruft, ist einzuwenden, dass alle europäischen Vorgaben umgesetzt sind, teilweise sogar weit über das gebotene Maß. Ein Beispiel - der zurzeit betriebene Missbrauch mit dem 2005 extrem weit gefassten Tatbestand des Menschenhandels. \232 Abs. 1, 2. Alternative StGB (der Grundtatbestand, besser Auffangtatbestand zu den Verbrechen des Menschenhandels - 2005 neu gestaltet) sieht eine Schutzaltersgrenze von 21 Jahren vor. Jede Handlung, die geeignet ist eine unter 21jährge Person dazu zu bringen sexuelle Handlungen gegen Entgelt vorzunehmen, kann danach von den Strafverfolgungsbehörden verfolgt werden. Die Praxis ist uneinheitlich und mehr oder weniger willkürlich. Der Grund für teilweise routinierte Strafverfolgungsmaßnahmen sind die leicht überprüfbaren Altersgrenzen. Da im Rotlichtmilieu der Pass der dort Tätigen häufig überprüft wird, können Anklagen/ Anträge auf Strafbefehle von der StA mehr oder weniger schematisch formuliert werden. Benutzt werden kann diese Strafnorm also immer dann gegen Bordellbetreiber, wenn man den Tatbestand der ausbeuterischen Prostitution nicht behaupten und schon gar nicht nachweisen kann (so in dem zurzeit betriebenen Verfahren gegen das "Colosseum“ in Augsburg, nachdem das LG Augsburg zuvor ein Strafverfahren wegen des Verdachts der ausbeuterischen Zuhälterei nicht eröffnet hatte).

Jugendliche und Kinder können in Fragen der Kontrolle der Sexualität nicht gleich behandelt werden, da Jugendliche ein allenfalls zu ihrem Schutz in Maßen begrenzbares Recht auf sexuelle Selbstbestimmung haben; schon gar nicht darf eine Erweiterung der strafrechtlichen Einschränkungen dieses Rechts durch Strafgesetze erfolgen, die rein modellplatonisch von Strafrechtswissenschaftlern ersonnen und begutachtet worden sind - ohne Blick auf die Bezugswissenschaften, welche die Entwicklungsbedingungen von Kindern und Jugendliche erforschen. Wenn man schon Jugendliche besser vor den Gefahren der Kommerzialisierung der Sexualität in hochmodernen Gesellschaften schützen will, dann nur unter Einbeziehung der Erkenntnisse der Sexual- und Sozialwissenschaften und der Erfahrungen der Jugendforschung und Jugendsozialarbeit. Eine sinnvolle Ergänzung der vorhandenen Jugendschutzbestimmungen hat sich daher auf einen besseren Schutz Jugendlicher bei der Verwendung ihrer personenbezogenen Daten zu konzentrieren. Nicht die Nutzer der Daten sind zu bestrafen, sondern diejenigen, welche ohne Einverständnis Bilder von Jugendlichen ins Netz stellen oder sich das Einverständnis von jungen Menschen erschleichen, um sie bloß zu stellen oder Geschäfte mit ihrem Bildmaterial zu machen. Dies setzt aber völlig andere Überlegungen voraus als sich die Gesetzgeber bislang gemacht haben.

\section{Fußnote:}

Der Bundestag hat die gesetzlichen Änderungen am 20.6.2008 in 3. Lesung beschlossen.

Minderjährigenschutz im Sexualstrafrecht

\begin{tabular}{|c|c|c|c|c|c|}
\hline \multirow{2}{*}{ Delikt } & & \multicolumn{4}{|c|}{ Geschützt werden Kinder/ Jugendliche } \\
\hline & & bis 14 Jahre & bis 16 Jahre & bis 18 Jahre & bis 21 Jahre \\
\hline & Kinderschutz & & & & \\
\hline \begin{tabular}{|l|l}
$\mathbb{S} 176,176 \mathrm{a}, 176 \mathrm{~b}$ StGB \\
\end{tabular} & Verbot sexueller Handlungen mit Kindern & $\bar{x}$ & & & \\
\hline & & & & & \\
\hline B. & Jugendschutz & & & & \\
\hline $\begin{array}{l}\text { \$ } 174 \mathrm{I} \text { Nr. } 1 \text { StGB } \\
\end{array}$ & in Abhängigkeitsverhältnissen & & $\mathrm{x}$ & & \\
\hline \$ $174 \mathrm{I} \mathrm{Nr}$. 2,3 StGB & Missbrauch in Abhängigkeitsverhältnissen & & & $\bar{x}$ & \\
\hline \$ 182 StGB & Ausnutzen einer Zwangslage & & $\mathrm{x}$ & & \\
\hline$\$ 180$ I StGB & Kuppelei (Vorschub leisten) & & $\mathrm{x}$ & & \\
\hline$\$ 180$ III StGB & Kuppelei bei Abhängigen & & & $\mathrm{x}$ & \\
\hline$\$ 180$ II StGB & Bestimmen zur entgeltlichen sexuellen Handlung & & & $\mathrm{x}$ & \\
\hline$\$ 182 \mathrm{StGB}$ & Sexueller Missbrauch Jugendlicher durch Erwachsene & & & $\mathrm{x}$ & \\
\hline & & & & & \\
\hline C. & Pornographie & & & & \\
\hline$\$ 176$ a III StGB & Persönlichkeitsschutz (Verbrechen) & $\mathrm{x}$ & & & \\
\hline$\$ 184$ I StGB & Jugendschutz & & & $\mathrm{x}$ & \\
\hline$\$ 184 \mathrm{~b}$ StGB & Konsumentenbestrafung & & $\mathrm{xxxxxxx}$ & $\overline{x x x x x x x}$ & \\
\hline D. & Prostitution und ähnliches Verhalten & & & & \\
\hline \$ 180 I StGB & Kuppelei (Vorschub leisten) & & $\mathrm{x}$ & & \\
\hline$\$ 180$ III StGB & Kuppelei bei Abhängigen & & & $\mathrm{x}$ & \\
\hline$\$ 180$ II StGB & Bestimmen zur entgeltlichen sexuellen Handlung & & & $\mathrm{x}$ & \\
\hline \$ 180a II Nr. 1 StGB & Verbot der Wohnungsgewährung & & & $\mathrm{x}$ & \\
\hline$\$ 182 \mathrm{StGB}$ & sehr weit gefasste Freierbestrafung & & & $\mathrm{xxxxxxx}$ & \\
\hline & & & & & \\
\hline E. & Verbot der Prostitution bei jungen Menschen & & & & \\
\hline$\$ 232$ I 2 StGB & Einschränkung der Erlaubnis der Prostitution (nach ProstG) & & & & $\mathrm{x}$ \\
\hline$\$ 233$ I 2 StGB & Gewalt & & & & $x$ \\
\hline$\$ 233 \mathrm{a}$ II Nr. 1 StGB & Vorverlagerung des strafrechtlichen Schutzes bei & $\mathrm{x}$ & & & \\
\hline & kindlichen Opfern & & & & \\
\hline
\end{tabular}

